\title{
BMJ Open Fetal Alcohol Spectrum Disorder resources for educators working within primary school settings: a scoping review protocol
}

\author{
Briana Lees (D) , ${ }^{1}$ Elizabeth J Elliott (D) ,,3 Steve Allsop (D) , ${ }^{4}$ Sue Thomas, ${ }^{5}$ \\ Julia Riches, ${ }^{1}$ Smriti Nepal (D) , ${ }^{1}$ Lauren J Rice (D) , ${ }^{3,6}$ Nicola Newton (D) , ${ }^{1}$ \\ Louise Mewton (D) , ${ }^{7}$ Maree Teesson (D) , ${ }^{1}$ Lexine A Stapinski (D) ${ }^{1}$
}

To cite: Lees B, Elliott EJ, Allsop S, et al. Fetal Alcohol Spectrum Disorder resources for educators working within primary school settings: a scoping review protocol. BMJ Open 2021;11:e045497. doi:10.1136/ bmjopen-2020-045497

- Prepublication history and additional supplemental material for this paper are available online. To view these files, please visit the journal online (http://dx.doi.org/10.1136/ bmjopen-2020-045497).

Received 02 October 2020 Revised 17 February 2021 Accepted 12 March 2021

Check for updates

(C) Author(s) (or their employer(s)) 2021. Re-use permitted under CC BY-NC. No commercial re-use. See rights and permissions. Published by BMJ.

For numbered affiliations see end of article.

Correspondence to

Briana Lees;

briana.lees@sydney.edu.au

\section{ABSTRACT}

Introduction Many children affected by Fetal Alcohol Spectrum Disorder (FASD) exhibit neurocognitive delays that contribute to secondary consequences, including a disrupted school experience. Educators often have limited knowledge or experience in the identification, referral, management and accommodation of students with FASD. Effective resources and tools for educators are crucial to ensure these students are supported in their ongoing learning, development and school participation. This scoping review aims to identify and evaluate resources for educators that aid in the identification, management, or accommodation of students with FASD.

Methods and analysis A search will be conducted in 9 peer-reviewed and 11 grey literature databases, Google search engine, two app stores and two podcast streaming services (planned search dates: November 2020 to February 2021). Relevant experts, including researchers, health professionals and individuals with lived experience of FASD, will be contacted in February and March 2021 to identify additional (including unpublished) resources. Resources will be selected based on registered, prespecified inclusion-exclusion criteria, and the quality of included resources will be critically appraised using a composite tool based on adaptions of the National Health and Medical Research Council FORM Framework and the iCAHE Guideline Quality Checklist. Relevant experts will also be requested to provide feedback on included resources.

Ethics and dissemination Ethical approval for this scoping review was obtained from the University of Sydney Human Research Ethics Committee (2020/825). Results of the review will be disseminated through a peer-reviewed publication, conference presentations, and seminars targeting audiences involved in the education sector. Trial registration Open Science Framework: osf.io/73pjh.

\section{INTRODUCTION}

Fetal Alcohol Spectrum Disorder (FASD) is a diagnostic term that describes the continuum of effects resulting from prenatal alcohol exposure, including neurodevelopmental, physical, emotional and behavioural
Strengths and limitations of this study

- This scoping review will be the first to collate a broad range of Fetal Alcohol Spectrum Disorder (FASD)-related resources relevant for educators to effectively recognise and respond to students with FASD.

- The scoping review will adhere to an established six-stage review framework.

- Following the review, access links for the identified high-quality resources will be made available in an online portal, and based on identified gaps, new resources will be developed to support educators.

- The review will be limited to FASD resources despite clinical presentation overlap with other neurodevelopmental disorders.

- The strength of the conclusions and the use of the scoping review will be limited by the quality of available resources identified.

consequences. ${ }^{1}$ The estimated global prevalence of FASD among children is $0.77 \%,{ }^{2}$ placing FASD as the leading preventable cause of non-genetic developmental disabilities. ${ }^{3}$ Factors such as dose and exposure patterns, as well as biological, genetic, epigenetic and accompanying environmental factors contribute to the significant variability in the range and magnitude of adverse outcomes associated with prenatal alcohol exposure. ${ }^{45}$

Many children affected by FASD exhibit neurocognitive delays, including attention and executive functioning difficulties, poor memory, impaired reasoning and judgement, learning disabilities and sensory processing and spatial awareness problems. ${ }^{6}$ These delays contribute to secondary outcomes; for example, $94 \%$ of individuals with FASD experience at least one mental health problem and $70 \%$ have been suspended, expelled or dropped out of school. ${ }^{7}$ Additionally, some 
children with FASD have experienced deprivation or neglect resulting from parental mental health, substance use or social problems. ${ }^{8-10}$ These factors in combination contribute to risk of a disrupted school experience for children affected by FASD, particularly if they do not receive appropriate support and intervention.

Adding to these challenges, children with FASD often attend schools where educators have limited knowledge or expertise in the identification, assessment, management or mentorship of students with developmental, learning, social and behavioural problems associated with FASD. ${ }^{11}$ To ensure the appropriate referral and management of such students, and to support their ongoing learning, development and school participation, educators must be equipped with effective tools. Previous reviews have synthesised FASD identification tools and behavioural management resources. In 2008, an expert panel review summarised Canadian screening tools for FASD and identified one tool which had potential for use within the school setting. ${ }^{12}$ However, a review and comparison of available assessment tools developed outside of Canada and that are suitable for the education sector is not available. Systematic and scoping reviews have also synthesised health, behavioural and educational interventions for individuals with FASD. ${ }^{13-16}$ These reviews included student-focused interventions that aim to address neurodevelopmental delays, self-regulation and social skills. Two of the reviews described professional development training programmes for educators that include management techniques for use with students with FASD. ${ }^{13} 16$ While the identified programmes are important, educators require a broader suite of resources to adequately manage and mentor these students, including access to FASD guidelines and policies that are relevant to the school setting, professional development and information-based tools such as factsheets, workshops and webinars, and outlines of effective strategies for managing behaviour within the classroom. To date, there has been no review which has comprehensively collated FASD-related resources to equip educators to effectively recognise and respond to students with FASD.

To meet this need, we will conduct a scoping review to identify and evaluate the quality of resources suitable for the school setting. We seek to comprehensively identify resources which assist educators in identifying students with developmental, learning, social and behavioural problems consistent with FASD requiring medical referral, as well as tools to support ongoing learning, development and school participation for students with FASD. Although there is significant overlap in neurocognitive impairment, developmental delay, comorbidities and secondary outcomes among students with FASD and other neurodevelopmental disorders and brain injuries ${ }^{17-19}$ this review will be limited to resources which are explicitly relevant for, or designed to, educate, assist or build capacity among educators in identifying, managing or accommodating students with FASD. This review will focus on resources suitable for educators working with primary school-aged rather than secondary school-aged students. This is because early identification, diagnosis and intervention are important factors associated with reduced risk of long-term adverse outcomes for individuals with FASD, including disrupted school experience as well as troubles with the law, psychiatric hospitalisation, and drug and alcohol problems. ${ }^{1820}$

\section{METHODS}

\section{Protocol design}

The scoping review is informed by the Arksey and O'Malley framework, ${ }^{21}$ which has been further developed by Levac and colleagues ${ }^{22}$ and the Joanna Briggs Institute (JBI).$^{23}$ This framework organises the review process in six stages: (1) identifying the research questions; (2) identifying relevant resources; (3) resource selection; (4) charting the data; (5) collating, summarising and reporting the results; and (6) consultation with key stakeholders to identify additional resources and gain feedback on results. Below, we provide methodological details of each pre-registered stage (osf.io/73pjh).

\section{Stage 1: identifying the research questions}

The primary research question of this scoping review is: What resources and/or guidelines are available for educators that (1) enhance identification, assessment and referral of students with developmental, learning, social and behavioural problems consistent with FASD, and (2) aid management or accommodation of FASDrelated symptoms and behaviours in a school setting? Secondary research questions include: (1) What is the quality of existing resources in terms of evidence base, impact and utility, generalisability, applicability, credibility, availability, ease of use and currency? and (2) Are resources designed to improve the management or accommodation of developmental, learning, social, and behavioural problems associated with FASD? If so, what are the effective components?

\section{Stage 2: identifying relevant resources Selection criteria}

As outlined in the JBI manual for evidence synthesis, ${ }^{23}$ the source of information will include any existing literature, such as, but not limited to, primary research studies, systematic reviews or meta-analyses, guidelines and policies, books, programmes and professional development and information-based resources (eg, factsheets, videos, podcasts, apps, websites). Herein, all sources and types of information are referred to as resources. Resources will be included in the scoping review if they meet the following inclusion criteria:

1. Population: educators (ie, principals, teachers, education assistants, school psychologists, allied health teams and nurses, Indigenous Liaison Officers or other school staff) who interact with and support primary school-aged children with FASD in a school setting.

2. Concept: 
i. Identify and evaluate resources relevant for, or designed to, educate, assist or build capacity among educators in recognising and referring students with developmental, learning, social, and behavioural problems consistent with FASD; and,

ii. Identify and evaluate resources for relevant educators that are designed to aid in the management or accommodation of students with FASD indicators or diagnosis, including resources that provide education and mentoring to these students.

3. Context: International resources will be reviewed.

4. Time frame: any.

5. Resource accessibility: the resource must be currently publicly available for educators, including free resources and those available for purchase. Current availability will be determined by online searching, recent publications, or contact with resource creators.

6. Language: English.

\section{Search strategy}

Resources will be identified by searching the peerreviewed and grey literature (ie, published or unpublished resources in non-commercial form), Google search engine, app stores, podcast streaming services and consultations with relevant FASD experts (see table 1). The search will be conducted using an iterative process by one member of the research team. First, an initial search of at least one bibliographic database and one grey literature source will be conducted to identify keywords and search terms used in the title, abstract, description and/or index terms of resources (note: this step has been completed). Next, selected databases, grey literature, the Google search engine, app stores and podcast streaming services will be searched using the keywords defined in step 1, summarised below and detailed in the online supplemental file. This stage will take place

\section{Table 1 Information sources to be searched}

PsycINFO, PubMED, Scopus, Web

Peer-reviewed of Science, ERIC, Cochrane Library, databases MEDLINE, EMBASE, CINAHL

\begin{tabular}{|c|c|}
\hline Grey literature & $\begin{array}{l}\text { Google advanced search*, FASD Hub, } \\
\text { NOFASD, HealthlnfoNet, Australian } \\
\text { Department of Education websites, } \\
\text { Australian Department of Health websites, } \\
\text { Education Services Australia, National } \\
\text { Organisation for FASD (UK), The National } \\
\text { Organization for Fetal Alcohol Syndrome } \\
\text { (US), Children's Healthcare Canada, The } \\
\text { Provincial Outreach Program for Fetal } \\
\text { Alcohol Spectrum Disorder }\end{array}$ \\
\hline Apps & Apple App Store, Google Play \\
\hline Podcasts & Apple Podcast, Spotify \\
\hline Consultations & FASD experts \\
\hline
\end{tabular}

${ }^{*}$ Guided by previous scoping reviews, ${ }^{27}$ the screening of the Google advance search will be limited to the first five pages of results (20 results per page; total $=100$ ). from November 2020 to February 2021. International FASD experts will then be contacted via email or phone in February and March 2021 and asked to provide any relevant resources for educators (including unpublished resources) and suggest any colleagues or other individuals who might have knowledge regarding such resources. Finally, all relevant resources will be imported into reference management software and an excel spreadsheet, where duplicates will be removed.

\section{Search terms}

In step 1 of the search strategy, the following search terms were entered into MEDLINE, PsycINFO, EMBASE, ERIC and Google: ('fetal alcohol spectrum disorder' OR 'fetal alcohol syndrome') AND (intervention OR program) AND (review OR 'systematic review' OR 'scoping review'). Relevant review articles were retrieved and examined for search terms ${ }^{13-16}$ Based on this exploratory search phase, the search strings for each database, website, app store, and podcast streaming service were finalised.

FASD-related search terms include: 'fetal alcohol spectrum disorder', 'fetal alcohol syndrome', 'alcohol related neurodevelopmental disorder', 'alcohol related birth defects', 'prenatal alcohol exposure' and 'alcohol use during pregnancy'. Resource-related terms include: 'program', 'intervention', 'management', 'guidelines', 'guide', 'policy', 'factsheet', 'information sheet', 'booklet', 'video', 'screening tool', and 'assessment'. Setting-related terms include: 'school', 'school based', 'teacher' and 'educator'. Where possible, the same search terms and fields will be used in database searches, using the websites' search function. When the capacity of the website does not allow for multiple search terms, singular phrases will be searched (eg, 'fetal alcohol spectrum disorder'). Searches for alternate spelling will also be conducted (eg, fetal vs foetal).

\section{Stage 3: resource selection}

Once resources have been imported into reference management software, a three-phase screening and selection process will be undertaken: (1) screen title/abstract/ description of resources for eligibility; (2) access data sources/obtain full-text versions of resources considered eligible in step 1 and further screen for eligibility; and (3) search for other relevant resources in the reference lists of identified materials and screen for eligibility by following steps 1-2.

One member of the research team will undertake step 1 , where a sample (ie, $20 \%$ ) of the retrieved resources will additionally be screened by a second team member to ensure consistent application of the eligibility criteria for inclusion in the review. Step 2 will be independently undertaken by two team members, with any disagreement resolved by a third member. One researcher will conduct the search for other relevant resources in step 3. For steps 1 and 2, Cohen's kappa will be calculated to assess the inter-rater agreement between the two reviewers. The process of study selection will be reported in the scoping 
Table 2 Draft of customised data charting tool for FASD resources

\begin{tabular}{ll}
\hline Category & Details \\
\hline Authors/developers & \\
Title &
\end{tabular}

Year of publication

Context Country of origin

Resource category Identification/assessment or management resource

Resource type

Screening and assessment measures of FASD, guidelines, interventions or programmes or information-based resources

Resource description $\begin{aligned} & \text { Delivery methods, lengths, } \\ & \text { objectives etc }\end{aligned}$

Target population Type of educator (eg, principals, teachers, education assistants, school psychologists, allied health teams and nurses or other school staff)

$\begin{array}{ll}\begin{array}{l}\text { Has the resource been } \\ \text { evaluated? If yes, provide } \\ \text { details }\end{array} & \begin{array}{l}\text { Sample size, sociodemographic } \\ \text { characteristics of sample, } \\ \text { reported outcomes, } \\ \text { effectiveness, impact, validation } \\ \text { (for screening measures) }\end{array} \\ \begin{array}{l}\text { Details on evidence base } \\ \text { of the resource }\end{array} & \begin{array}{l}\text { Evidence base of resource (eg, } \\ \text { formal evaluation or published } \\ \text { findings) or evidence base } \\ \text { that informed development of } \\ \text { resource }\end{array}\end{array}$

\section{Resource costs}

Other information

Reference/website

FASD, fetal alcohol spectrum disorder.

review using a Preferred Reporting Items for Systematic Reviews and Meta-Analyses (PRISMA) flow chart.

\section{Stage 4: Charting the data \\ Charting}

Information on each eligible resource will be charted using a customised data charting tool, developed for this review and informed by the JBI manual (table 2). ${ }^{23}$ Two team members will initially chart three resources to test the adequacy of the tool in extracting all relevant data and they will modify the tool as required. The remaining data will be charted by one team member. Data charting will involve an iterative process whereby the charting tool will be continually modified and updated as the team member charts each resource and identifies additional data of interest. ${ }^{23}$

\section{Quality appraisal}

The quality of included resources will be assessed using a composite of two critical appraisal tools: an adapted version of the National Health and Medical Research
Council (NHMRC) FORM framework ${ }^{24}$ and the iCAHE Guideline Quality Checklist. ${ }^{25}$ A composite tool will be used to ensure that all relevant domains in varying types of resources are assessed for quality. Resources will be evaluated on their evidence base, impact and utility, generalisability, availability, currency, ease of use, credibility and applicability (table 3 ). In line with the NHMRC FORM framework, each component will receive a score ranging from A (excellent) to D (poor). Based on individual component scores, an overall grade for each resource will be calculated. One member of the research team will appraise all resources and a second member will additionally appraise $20 \%$ of included resources to ensure consistent evaluation. Disagreements will be discussed by the two reviewers, and if any disagreement cannot be resolved, a third member of the research team will be consulted to arbitrate the decision.

\section{Stage 5: Collating, summarising and reporting the results}

The reporting of the scoping review will be informed by the PRISMA extension for Scoping Reviews Checklist. ${ }^{26}$ The results of the search, and each screening step, will be presented in a PRISMA flowchart. The findings from the charting and quality appraisal will be summarised and presented in table form.

\section{Stage 6: Consultation with key stakeholders to identify additional resources and seek feedback}

As outlined above, consultation will be conducted with FASD experts during stage 2 (simultaneously with online searching procedures) to identify additional resources relevant to the scoping review. After collating and summarising results, further consultation with FASD experts will occur during stage 6 to seek feedback on our results and to identify gaps in currently accessible resources for educators.

\section{Patient and public involvement}

Patients and the general public were not involved in development of this protocol. However, experts, including researchers, health professionals and individuals with lived experience of FASD, will be consulted during stages 2 and 6 of the scoping review in order to locate additional resources relevant for educators and to seek feedback on identified resources.

\section{Dissemination and ethics}

This scoping review will be the first to identify and evaluate a broad range of resources suitable for use in school settings to assist educators in identifying, managing, accommodating and mentoring primary school-aged students with FASD. This protocol reports a comprehensive, rigorous and transparent methodology that has received ethical approval from the University of Sydney Human Research Ethics Committee (2020/825).

A limitation of this protocol is the search strategy is restricted to FASD resources despite students with FASD exhibiting overlap in clinical presentation with other neurodevelopmental disorders. However, a broader focus 
Table 3 A composite quality appraisal tool based on an adaption of the NHMRC Evidence Statement Form, the NHMRC Body of Evidence Matrix and the iCAHE Guidelines Quality Checklist.

\begin{tabular}{|c|c|c|c|c|}
\hline & A & B & C & D \\
\hline Component & Excellent & Good & Satisfactory & Poor \\
\hline Evidence base & $\begin{array}{l}\text { Formally evaluated and } \\
\text { findings published }\end{array}$ & $\begin{array}{l}\text { No formal evaluation; } \\
\text { developed on the basis } \\
\text { of published findings } \\
\text { OR some testing among } \\
\text { endusers has been } \\
\text { conducted }\end{array}$ & $\begin{array}{l}\text { No reference to formal } \\
\text { evidence or testing; } \\
\text { developed by expert } \\
\text { consensus }\end{array}$ & $\begin{array}{l}\text { Developed on the basis } \\
\text { of personal opinion only }\end{array}$ \\
\hline Generalisability & $\begin{array}{l}\text { Relevant to one or more of the } \\
\text { target groups }\end{array}$ & $\begin{array}{l}\text { Some information relevant } \\
\text { for the target group }\end{array}$ & & $\begin{array}{l}\text { Not relevant for any of } \\
\text { the target groups }\end{array}$ \\
\hline Applicability & $\begin{array}{l}\text { Directly applicable to the } \\
\text { Australian context }\end{array}$ & $\begin{array}{l}\text { Applicable to the Australian } \\
\text { context with some caveats }\end{array}$ & & $\begin{array}{l}\text { Not applicable to an } \\
\text { Australian context/ } \\
\text { unsure }\end{array}$ \\
\hline Currency & $\begin{array}{l}\text { Resource is current/regularly } \\
\text { updated }\end{array}$ & $\begin{array}{l}\text { Resource is current but not } \\
\text { updated regularly }\end{array}$ & $\begin{array}{l}\text { Resource is not current } \\
\text { but contains up-to- } \\
\text { date information and } \\
\text { terminology ( } \geq 10 \text { years) }\end{array}$ & $\begin{array}{l}\text { Resource contains out } \\
\text { of date information and } \\
\text { terminology }\end{array}$ \\
\hline Ease of use & Easy to use or navigate & & & $\begin{array}{l}\text { Difficult to use or overly } \\
\text { complex }\end{array}$ \\
\hline Credibility & $\begin{array}{l}\text { University-based or } \\
\text { government funded, reputable } \\
\text { developers }\end{array}$ & & & $\begin{array}{l}\text { Developers or } \\
\text { organisation not } \\
\text { reputable }\end{array}$ \\
\hline
\end{tabular}

NHMRC, National Health and Medical Research Council.

on resources for other neurodevelopmental disorders would substantially widen the scope of the review and hinder our ability to directly address the primary and secondary research questions.

The results of this scoping review will be disseminated through a peer-reviewed publication, conference presentations and seminars targeting audiences involved in the education sector and FASD research. Access links for identified high-quality resources will be consolidated in an online portal, and based on identified gaps, new resources will be developed to support educators. It is expected that the scoping review will be completed and disseminated in 2021.

\section{Author affiliations}

${ }^{1}$ The Matilda Centre for Research in Mental Health and Substance Use, The University of Sydney, Camperdown, New South Wales, Australia

${ }^{2}$ Faculty of Medicine and Health, Discipline of Child and Adolescent Health, The University of Sydney, Sydney, New South Wales, Australia

${ }^{3}$ The Sydney Children's Hospitals Network, Westmead, New South Wales, Australia ${ }^{4}$ National Drug Research Institute, Curtin University, Perth, Western Australia, Australia

${ }^{5}$ Marulu Unit, Marninwarntikura Women's Resource Centre, Fitzroy Crossing, Western Australia, Australia

${ }^{6}$ The Brain and Mind Centre, The University of Sydney, Sydney, New South Wales, Australia

${ }^{7}$ Centre for Healthy Brain Ageing, University of New South Wales, Randwick, New South Wales, Australia
Twitter Briana Lees @brianalees

Contributors BL and LAS conceptualised the study and are the guarantors of the review. EJE, SA, ST, JR, SN, LJR, NN, LM and MT provided critical feedback on the study design and protocol. BL wrote the first draft of the manuscript and all authors read, revised and approved the final version. All authors agree to be accountable for all aspects of the work.

Funding Briana Lees, Elizabeth J Elliott, Maree Teesson and Lexine A Stapinski are funded by the Australian National Health and Medical Research Council (BL: GNT1169377; EJE: GNT1021480, GNT1110341; MT: GNT1041756, GNT1078407; LAS: GNT1132853). Lauren J Rice is funded by The lan Potter Foundation (\#31110414). Sue Thomas is funded by the National Indigenous Australians Agency. Competing interests None declared.

Patient consent for publication Not required.

Provenance and peer review Not commissioned; externally peer reviewed.

Supplemental material This content has been supplied by the author(s). It has not been vetted by BMJ Publishing Group Limited (BMJ) and may not have been peer-reviewed. Any opinions or recommendations discussed are solely those of the author(s) and are not endorsed by BMJ. BMJ disclaims all liability and responsibility arising from any reliance placed on the content. Where the content includes any translated material, BMJ does not warrant the accuracy and reliability of the translations (including but not limited to local regulations, clinical guidelines, terminology, drug names and drug dosages), and is not responsible for any error and/or omissions arising from translation and adaptation or otherwise.

Open access This is an open access article distributed in accordance with the Creative Commons Attribution Non Commercial (CC BY-NC 4.0) license, which permits others to distribute, remix, adapt, build upon this work non-commercially, and license their derivative works on different terms, provided the original work is properly cited, appropriate credit is given, any changes made indicated, and the use is non-commercial. See: http://creativecommons.org/licenses/by-nc/4.0/. 


\section{ORCID iDs}

Briana Lees http://orcid.org/0000-0002-9888-8794

Elizabeth J Elliott http://orcid.org/0000-0002-6501-5487

Steve Allsop http://orcid.org/0000-0003-1087-3027

Smriti Nepal http://orcid.org/0000-0002-6263-6763

Lauren J Rice http://orcid.org/0000-0002-2315-7698

Nicola Newton http://orcid.org/0000-0001-6305-2623

Louise Mewton http://orcid.org/0000-0002-7812-296X

Maree Teesson http://orcid.org/0000-0002-6744-463X

Lexine A Stapinski http://orcid.org/0000-0002-3656-4655

\section{REFERENCES}

1 Bower C, Elliott EJ, Group on behalf of the S. Report to the Australian government department of health: Australian guide to the diagnosis of fetal alcohol spectrum disorder (FASD) 2016.

2 Lange S, Probst C, Gmel G, et al. Global prevalence of fetal alcohol spectrum disorder among children and youth: a systematic review and meta-analysis. JAMA Pediatr 2017;171:948-56.

3 O'Leary C. Fetal alcohol syndrome : a literature review / prepared for the National Expert Advisory Committee on Alcohol. 50554210. Canberra, A.C.T: Commonwealth Department of Health and Ageing, 2002. https://trove.nla.gov.au/work/16825160?q\&versionld= 46500264

4 Loock C, Elliott E, Cox L. Fetal alcohol spectrum disorder. In: The Routledge Handbook of social work and addictive behaviors, 2020.

5 Lees B, Mewton L, Jacobus J, et al. Association of prenatal alcoho exposure with psychological, behavioral, and neurodevelopmental outcomes in children from the adolescent brain cognitive development study. Am J Psychiatry 2020;177:1060-72.

6 Mattson SN, Bernes GA, Doyle LR. Fetal alcohol spectrum disorders: a review of the neurobehavioral deficits associated with prenatal alcohol exposure. Alcohol Clin Exp Res 2019;43:1046-62.

7 Streissguth A, Barr H, Kogan K. Understanding the occurrence of secondary disabilities in clients with fetal alcohol syndrome (Fas) and fetal alcohol effects (FAE). Final report to the centers for disease control and prevention (CDC) 1996.

8 Streissguth AP, Bookstein FL, Barr HM, et al. Risk factors for adverse life outcomes in fetal alcohol syndrome and fetal alcohol effects. $J$ Dev Behav Pediatr 2004;25:228-38 https://pubmed.ncbi.nlm.nih.gov/ 15308923/

9 Hussong AM, Huang W, Curran PJ, et al. Parent alcoholism impacts the severity and timing of children's externalizing symptoms. $J$ Abnorm Child Psychol 2010;38:367-80.

10 Koponen AM, Kalland M, Autti-Rämö I. Caregiving environment and socio-emotional development of foster-placed FASD-children. Child Youth Serv Rev 2009;31:1049-56.

11 Millians MN. Educational needs and care of children with FASD. Curr Dev Disord Rep 2015;2:210-8 https://link.springer.com/article/

12 Goh YI, Chudley AE, Clarren SK, et al. Development of Canadian screening tools for fetal alcohol spectrum disorder. Can J Clin
Pharmacol 2008;15:e344-66 http://europepmc.org/article/med/ 18840921

13 Reid N, Dawe S, Shelton D, et al. Systematic review of fetal alcohol spectrum disorder interventions across the life span. Alcohol Clin Exp Res 2015;39:2283-95.

14 Premji S, Benzies K, Serrett K, et al. Research-based interventions for children and youth with a fetal alcohol spectrum disorder: revealing the gap. Child Care Health Dev 2007;33:389-97 https:// pubmed.ncbi.nlm.nih.gov/17584393/

15 Peadon E, Rhys-Jones B, Bower C, et al. Systematic review of interventions for children with fetal alcohol spectrum disorders. BMC Pediatr 2009;9:35 https://www.ncbi.nlm.nih.gov/pubmed/19463198

16 Adebiyi BO, Mukumbang FC, Erasmus C. The distribution of available prevention and management interventions for fetal alcohol spectrum disorder (2007 to 2017): implications for collaborative actions, International Journal of environmental research and public health. Mdpi Ag 2019;16.

17 Mattson SN, Crocker N, Nguyen TT. Fetal alcohol spectrum disorders: neuropsychological and behavioral features. Neuropsychol Rev 2011;21:81-101.

18 Stevens SA, Nash K, Koren G, et al. Autism characteristics in children with fetal alcohol spectrum disorders. Child Neuropsychol 2013;19:579-87.

19 Lange S, Shield K, Rehm J, et al. Fetal alcohol spectrum disorder: neurodevelopmentally and behaviorally indistinguishable from other neurodevelopmental disorders. BMC Psychiatry 2019;19:322 https:// bmcpsychiatry.biomedcentral.com/articles/

20 McDougall S, Finlay-Jones A, Arney F, et al. A qualitative examination of the cognitive and behavioural challenges experienced by children with fetal alcohol spectrum disorder. Res Dev Disabil 2020;104:103683.

21 Arksey H, O'Malley L. Scoping studies: towards a methodological framework. Int J Soc Res Methodol 2005;8:19-32 https://www. tandfonline.com/doi/abs/

22 Levac D, Colquhoun H, O'Brien KK. Scoping studies: advancing the methodology. Implement Sci 2010;5:69.

23 Peters M, Godfrey C, McInerney P. Chapter 11: Scoping Reviews. In: JBI manual for evidence synthesis. JBI, 2020. https://wiki.jbi.global/ display/MANUAL/Chapter+11\%3A+Scoping+reviews

24 Hillier S, Grimmer-Somers K, Merlin T, et al. Form: an Australian method for Formulating and grading recommendations in evidencebased clinical guidelines. BMC Med Res Methodol 2011;11:23 https://bmcmedresmethodol.biomedcentral.com/articles/

25 Grimmer K, Dizon JM, Milanese S, et al. Efficient clinical evaluation of guideline quality: development and testing of a new tool. BMC Med Res Methodol 2014;14:63 https://bmcmedresmethodol. biomedcentral.com/articles/

26 Tricco AC, Lillie E, Zarin W, et al. PRISMA extension for scoping reviews (PRISMA-ScR): checklist and explanation. Ann Intern Med 2018;169:467-73.

27 Bassett-Gunter R, Angevaare K, Tomasone J, et al. A systematic scoping review: resources targeting the training and education of health and recreation practitioners to support physical activity among people with physical disabilities. Disabil Health J 2019;12:542-50 https://pubmed.ncbi.nlm.nih.gov/31231020/ 